\title{
ICSH recommendations for measurement of erythrocyte sedimentation rate
}

\author{
International Council for Standardization in Haematology (Expert Panel on Blood \\ Rheology)
}

\section{Introduction}

Tests of erythrocyte sedimentation provide a measure of the acute phase response to inflammatory disease ${ }^{1}$ and reflect the sedimentation of red cells in autologous plasma. The term erythrocyte sedimentation rate (ESR) is retained because of traditional usage, although a single measurement after 60 minutes is not a rate; in recording the ESR it is assumed that the reading is made at 60 minutes unless a different time is specified. Sedimentation is accelerated by an increase in the plasma concentration of acute phase proteins of large molecular size but sedimentation is also accelerated by anaemia which may or may not be part of inflammatory disease. The ESR may therefore reflect both the hyperproteinaemia and the anaemia of inflammatory disease and differs from tests, such as plasma viscosity, which reflect only the protein component of the acute phase response.

The increased mobility of patients and the benefits to laboratories of sharing their experience has led to the need for measurements between laboratories to be comparable. This can be achieved by using a reference method. ICSH has defined this as an exactly described technique which, in the opinion of an Expert Panel, provides sufficiently accurate and precise measurement for it to be used to assess the validity of other such laboratory methods. The original ICSH reference method for measuring the $\mathrm{ESR}^{2}$ was based on the methodology of Fåhraeus ${ }^{3}$ and Westergren ${ }^{4}$ using diluted blood (4 vols blood plus 1 vol citrate) in open ended glass tubing of 300 $\mathrm{mm}$ in length, mounted vertically in a rack or stand. Modifications of these specifications, in particular the use of undiluted blood, are now recommended as the basis of a new ICSH reference method.

Recent developments, including biohazard awareness and difficulty in obtaining equipment to perform the reference method, have prompted ICSH to introduce a standardised method as an alternative to, and potential replacement for, the reference method.

For working (routine) methods, ICSH now recommends specifications for selected methods. These are procedures whose reliability has been verified against the reference or standardised method and which minimise the biohazard risk of the test procedure.

\section{ICSH reference method}

In 1988 ICSH proposed, for purposes of intermethod comparability, an ESR per- formed on undiluted blood samples of haematocrit of 0.35 or less under standardised conditions in a Westergren open ended glass pipette that meets ICSH specifications. ${ }^{1}$ These undiluted blood samples are anticoagulated with EDTA (dilution less than 1\%) but not diluted with citrate anticoagulant. This method will now be recognised by ICSH as its reference method. The reference method, and the standardised method described below, exist to allow users to prepare ESR reference material for verification or quality control purposes in their own laboratories. The results from both methods should be expressed as ESR = (undiluted) $\times$ $\mathrm{mm}$. For comparison with the traditional method using diluted blood, a correction formula ${ }^{1}$ can be applied: diluted blood ESR mm $=($ undiluted blood ESR $\mathrm{m} \times 0 \cdot 86)-12$.

\section{ICSH standardised method}

AIM

The ICSH standardised method should be directly comparable with and traceable to the ICSH reference method and used as an alternative to it for verification or quality control, or both, of working (routine) methods.

\section{BLOOD SAMPLE}

Blood of haematocrit (or PCV) of 0.35 or less should be obtained by clean venepuncture over a maximum period of 30 seconds and without excessive venous stasis. Blood of higher haematocrit should not be used because reproducibility of sedimentation may be poor in narrow tubes. A manual or vacuum extraction venepuncture technique can be used and the blood should be taken into EDTA anticoagulant (dilution less than 1\%) without further dilution in citrate. The ESR should be set up within 4 hours of venepuncture.

\section{MIXING OF BLOOD SAMPLE}

Mixing of blood with EDTA anticoagulant $(1.5 \mathrm{mg} / \mathrm{ml} \mathrm{blood})$ is necessary at the time of venepuncture, but further mixing immediately before the ESR test is set up is critically important for reproducibility. For standard tubes $(10-12 \mathrm{~mm} \times 75 \mathrm{~mm}$ containing $5 \mathrm{ml}$ blood and with an air bubble comprising at ieast $20 \%$ of the tube volume) there should be a minimum of eight complete inversions $\left(180^{\circ} \times 2\right)$ with the air bubble travelling from end to end of the tube. Mixing must not cause haemolysis. Non-standard tubes, particularly when narrower, may require more than eight inversions and the required
Birmingham, Birmingham

Accepted for publication 26 August 1992 
number should be determined. Mixing should be continued until immediately before the ESR pipette is filled at the start of the test.

\section{SEDIMENTATION PIPETTE SPECIFICATIONS}

The pipette (tubes) should be colourless, circular, and of sufficient length to give a 200 $\mathrm{mm}$ sedimentation scale. The scale may be marked on the pipette or separately and should comprise clearly marked lines at $1 \mathrm{~mm}$ intervals and be numbered from 200 at the bottom up to 0 at appropriate intervals. If separate from the pipette, the scale must be part of a pipette holding device that ensures precise and reproducible alignment of the pipette and scale. If reading of the pipette is optico-electronic rather than visual, a marked scale is unnecessary.

The bore of sedimentation pipettes for the Westergren ESR has traditionally been 2.55 $\mathrm{mm}+/-0.15 \mathrm{~mm} .{ }^{5}$ ICSH now recommends that the pipette diameter should be not less than $2.55 \mathrm{~mm}$ (no upper limit is specified but the volume of blood required should be minimised). The bore should be constant (within $5 \%$ ) throughout its length and the interior of the pipette should be circular (difference between long and short axes not exceeding $0.1 \mathrm{~mm}$ ).

The ESR pipette should be disposable. Glass or plastic may be used but, if plastic, should not show adhesive properties towards blood cells and should not release plasticisers that affect blood or alter sedimentation. If a mould release agent is used in the manufacturing process, this must similarly not affect blood or alter sedimentation.

The pipette should be filled with blood to a height of at least $200 \mathrm{~mm}$. Adjustment of the blood column or scale should be possible to allow correction for slight variation in the nominal volume and ensure an initial reading of zero. During the sedimentation period, and during subsequent disposal, the system must prevent blood spillage or aerosol generation.

\section{PIPETTE HOLDING DEVICE}

The pipette should be held vertical (confirmed by a plumb line or equally effective device), protected from direct sunlight, draughts and vibration, and kept at a constant temperature $\left(+/-1^{\circ} \mathrm{C}\right)$ within the range $18-25^{\circ} \mathrm{C}$ during the period of sedimentation.

\section{EXPRESSING THE RESULT}

This should be recorded as the sedimentation occurring at 60 minutes from the beginning of the test and expressed as: ESR (undiluted) $=\times \mathrm{mm}$ (as for reference method above).

COMPARABILITY BETWEEN ICSH STANDARDISED AND ICSH REFERENCE METHODS

This procedure requires analysis of reference material (fresh human blood) on which the ESR has been determined by the ICSH reference method. The standardised method should be verified by comparison with the
ICSH reference method over a range of ESR values of 15-105 $\mathrm{mm}$. In this comparison $95 \%$ of the differences should be $5 \mathrm{~mm}$ or less, with the larger differences associated with higher ESR values.

\section{ICSH selected methods}

AIM

ICSH wishes to promote the use of ESR methodology that minimises biohazard risk; it is especially important to avoid blood spillage or aerosol generation during the test procedure. The purpose of designating working (routine) methods as ICSH selected is primarily to specify criteria that will allow meaningful comparison of results between laboratories. ICSH also considers that such criteria will encourage development of new methods of low biohazard risk that give comparable results with those of the ICSH reference method. A manufacturer who wants a working method to be recognised as $\mathrm{ICSH}$ selected should undertake a study of comparability with either the ICSH reference method or the ICSH standardised method. A similar study should be performed by an independent expert. The studies should be documented and readily available for scrutiny on request to the manufacturer.

For verification or quality control of an ICSH selected method in routine use, comparability checks against the ICSH standardised method are recommended.

\section{BLOOD SAMPLE}

Blood should be obtained by clean venepuncture over a maximum period of 30 seconds and without excessive venous stasis. A manual or vacuum extraction venepuncture technique can be used. The ESR test should be set up within 4 hours of venepuncture. Blood samples can be stored for more than 4 hours at $4{ }^{\circ} \mathrm{C}$, but any such longer period of storage must be validated and the evidence be available from the manufacturer for scrutiny. If certain types of blood sample-from cases of hyperlipidaemia or hyperbilirubinaemia, for example-are unsuitable for testing, this should be stated.

For blood samples that are diluted at venepuncture, 4 vols of blood may be taken directly into 1 vol of sterile sodium citrate anticoagulant-diluent. Vacuum tubes and tubes containing liquid anticoagulant for this purpose have a finite storage life which should be carefully defined by the manufacturer. Storage conditions must also be clearly specified. Alternatively, the blood may be taken first into a primary anticoagulant (EDTA) that does not cause significant dilution $(<1 \%)$ of plasma protein, followed by dilution in sterile sodium citrate. For the above purposes, the concentration of trisodium citrate dihydrate $\left(\mathrm{Na}_{3} \mathrm{C}_{6} \mathrm{H}_{5} \mathrm{O}_{7} .2 \mathrm{H}_{2} \mathrm{O}\right)$ should be within the range $0 \cdot 10-0.136 \mathrm{~mol} / \mathrm{l}$. This solution should be discarded if it becomes turbid; if kept in a reusable container, particular care must be taken to remove all traces of any detergent used for cleaning the 
container. If an alternative anticoagulant, diluent (such as saline), or dilution factor is used, comparability of the result with that obtained using the ICSH standardised (or reference) method should be documented and readily available from the manufacturer for scrutiny.

\section{MIXING OF BLOOD WITH ANTICOAGULANT- DILUENT}

Immediately before the ESR test is set up, the blood sample should be mixed as specified for the standardised method-at least eight complete inversions $\left(180^{\circ} \times 2\right)$ for a $10-12 \mathrm{~mm}$ $\times 75 \mathrm{~mm}$ blood container and more inversions if the interior diameter of the container is smaller. If the working method incorporates an automatic mixing device, its effectiveness should be validated by the manufacturer.

\section{SEDIMENTATION PIPETTE SPECIFICATIONS}

Pipette (tube) dimensions are not specified for ICSH selected methods but comparability of the test result with the ICSH standardised (or reference) method must be validated using the protocol described below. In the interests of safety, it is preferable that the pipette be disposable but, if reused, special attention must be paid to cleaning, with removal of all contaminants and an appropriate verification check made thereafter. The tube may be made of plastic or glass but, if plastic, should not show adhesive properties towards blood cells and should not release plasticisers that affect blood or alter sedimentation. Comparability data with the ICSH standardised (or reference) method should be readily available from the manufacturer.

\section{PIPETTE HOLDING DEVICE}

$A$ rack or stand should be provided to hold the sedimentation pipettes motionless. If not held vertical, the angle of incline should be specified and comparability of the result with that of the ICSH standardised (or reference) method should be validated. The pipettes should be protected from direct sunlight, draughts and vibration, and be maintained at a constant temperature $\left( \pm 1^{\circ} \mathrm{C}\right)$ within the range $18-25^{\circ} \mathrm{C}$ for the duration of the test. If applicable, adjustment of the blood column to zero should be possible to correct for slight deviation in the nominal volume and ensure an initial reading of zero.

\section{RECORDING THE END POINT}

The traditional Westergren method established that the end point should be read at 60 minutes. However, some systems allow readings at other times, which currently range from 20 to 120 minutes, or at multiple intermediate times (every 5 minutes, for example. The clinical usefulness of these alternative times is yet to be evaluated. At present, ICSH recommends that measurement be made at 60 minutes or normalised to 60 minutes.

\section{EXPRESSION OF RESULT}

The result should be expressed as: $\mathrm{ESR}=x$ $\mathrm{mm}$ (where $1 \mathrm{~mm}$ for diluted blood is equivalent to $1 \mathrm{~mm}$ for undiluted blood at $60 \mathrm{~min}$ utes according to the ICSH reference or standardised method; see reference method for correction formula for lack of dilution in reference/standardised methods). If an alternative method of recording the end point is used and the result is expressed as Westergren equivalent units, this must be clearly stated. If the result is adjusted mathematically for initial height of the blood column, haematocrit, ambient temperature, or duration of sedimentation, the method of adjustment should be described. The ESR result for the working method, whether requiring mathematical correction or not, should therefore be expressed so as to achieve comparability with the ICSH reference method.

\section{VERIFICATION}

Verification of any working (routine) ESR method using diluted blood should be performed against the ICSH standardised method at a rate determined by the laboratory's standard operating procedure and especially when a new batch of tubes or fresh stock of citrate is used. Verification against the ICSH standardised method, which is performed on undiluted blood, will detect errors in the volume or quality of the diluent and in the adequacy of mixing the diluent with blood in the working method. The ICSH standardised method can therefore also be used for quality control purposes.

To obtain blood for the verification exercise it may be convenient to select a patient EDTA sample of haematocrit of 0.35 or less that contains an adequate residue of blood, after all other tests are completed, and has an increased ESR value (range $15-105 \mathrm{~mm}$ ) as known from testing or as judged by clinical details or the extent of sedimentation after the sample has stood undisturbed for 30-60 minutes. The EDTA blood in the tube should then be mixed by at least eight complete inversions. After filling the pipette of the standardised method, another aliquot of blood from the same, or a duplicate, EDTA sample should be analysed by the laboratory's working method after dilution (4 vols blood plus 1 vol citrate).

If the blood sample for the working ESR method was taken by venepuncture directly into a ESR tube containing citrate, or if such a dilution was performed in the laboratory, the blood sample for the ICSH standardised ESR should be taken from a separate EDTA sample without dilution. This sample is usually available because a routine blood count is normally requested in parallel with the ESR. This blood sample should again have an ESR value between 15-105 $\mathrm{mm}$ and a haematocrit of 0.35 or less.

If a manufacturer wishes to demonstrate comparability of a new working method with the ICSH standardised (or reference) 
method, the verification exercise may be performed using normal blood with added fibrinogen to achieve an adequate range of increased ESR values (see protocol below). This verification should be followed by a similar exercise, in collaboration with an external clinical laboratory, using patients' blood samples. The results for both exercises should be documented and readily available for scrutiny on request to the manufacturer.

Verification of a working method is achieved if $95 \%$ of the results obtained fall within the limits shown in the table.

\section{REFERENCE VALUES}

Reference values should be established locally in accordance with the ICSH recommendation on reference values ${ }^{67}$ and expressed as for diluted blood (see selected method above). In view of the progressive rise in ESR with age, separate values should be established for each decade of adult life in men and women. Several other clinical variables influence the ESR and may therefore affect physiological reference values: haemoglobin concentration, medication, menstrual cycle, pregnancy, and smoking.

\section{MICROMETHODS}

Micromethods may be introduced for use in children or to reduce the draw volume for adult patients. Documented evidence of comparability with the ICSH reference or standardised method must be readily available from the manufacturer.
Protocol for evaluation of working ESR methods against the ICSH reference or ICSH standardised method

This protocol is based on ICSH recommendations for type testing equipment and apparatus used for haematological analysis, ${ }^{6} \mathrm{ICSH}$ guidelines on selection of laboratory tests for monitoring the acute phase response, ${ }^{1}$ ICSH recommendations for measuring the ESR of human blood, ${ }^{5}$ and this document.

In this protocol, the ESR equipment which is the subject of the evaluation will be referred to as the "test system". The reference method is that of $\mathrm{ICSH}^{1}$ using Westergren type glass tubes without anticoagulant diluent. The ICSH standardised method is as described in this document for undiluted blood.

\section{PRELIMINARY}

This is general information provided by the manufacturer and confirmed when the test system is installed in the evaluation laboratory. It should include the following:

1 Brand name and model, manufacturer, and distributor.

2 Suggested local price and cost of maintenance contract

3 Terms of guarantee

4 Overall dimensions and bench area requirement

5 Details of electrical supply and other necessary services, computer and robotic interface requirements, and requirement for waste disposal

6 Instruction manual giving principles of operation, degree of automation, data presen-

ESR values ( $\mathrm{mm}$ ) for verification of comparability of working (routine) method with ICSH standardised method

\begin{tabular}{|c|c|c|c|c|c|}
\hline $\begin{array}{l}\text { Standardised } \\
\text { Method }^{\star}\end{array}$ & $\begin{array}{l}\text { Working } \\
\text { Method } \\
\text { Limitst }\end{array}$ & $\begin{array}{l}\text { Standardised } \\
\text { Method }^{\star}\end{array}$ & $\begin{array}{l}\text { Working } \\
\text { Method } \\
\text { Limitst }\end{array}$ & $\begin{array}{l}\text { Standardised } \\
\text { Method }^{\star}\end{array}$ & $\begin{array}{l}\text { Working } \\
\text { Method } \\
\text { Limitst }\end{array}$ \\
\hline $\begin{array}{l}15 \\
16 \\
17 \\
18 \\
19 \\
20 \\
21 \\
22 \\
23 \\
24 \\
25 \\
26 \\
27 \\
28 \\
29 \\
30 \\
31 \\
32 \\
33 \\
34 \\
35 \\
36 \\
37 \\
38 \\
39 \\
40 \\
41 \\
42 \\
43 \\
44\end{array}$ & $\begin{array}{r}3-13 \\
4-14 \\
4-15 \\
4-15 \\
5-16 \\
5-17 \\
6-17 \\
6-18 \\
6-19 \\
7-19 \\
7-20 \\
8-21 \\
8-21 \\
9-22 \\
9-23 \\
10-24 \\
10-25 \\
11-25 \\
11-26 \\
12-27 \\
12-28 \\
13-29 \\
13-30 \\
14-30 \\
14-31 \\
15-32 \\
15-33 \\
16-34 \\
17-35 \\
17-36\end{array}$ & $\begin{array}{l}45 \\
46 \\
47 \\
48 \\
49 \\
50 \\
51 \\
52 \\
53 \\
54 \\
55 \\
56 \\
57 \\
58 \\
59 \\
60 \\
61 \\
62 \\
63 \\
64 \\
65 \\
66 \\
67 \\
68 \\
69 \\
70 \\
71 \\
72 \\
73 \\
74\end{array}$ & $\begin{array}{l}18-37 \\
18-38 \\
19-38 \\
20-39 \\
20-40 \\
21-41 \\
22-42 \\
22-43 \\
23-44 \\
24-45 \\
24-46 \\
25-47 \\
26-48 \\
26-49 \\
27-50 \\
28-51 \\
29-52 \\
29-53 \\
30-54 \\
31-56 \\
32-57 \\
32-58 \\
33-59 \\
34-60 \\
35-61 \\
35-62 \\
36-63 \\
37-64 \\
38-65 \\
39-66\end{array}$ & $\begin{array}{r}75 \\
76 \\
77 \\
78 \\
79 \\
80 \\
81 \\
82 \\
83 \\
84 \\
85 \\
86 \\
87 \\
88 \\
89 \\
90 \\
91 \\
92 \\
93 \\
94 \\
95 \\
96 \\
97 \\
98 \\
99 \\
100 \\
101 \\
102 \\
103 \\
104 \\
105\end{array}$ & $\begin{array}{l}40-68 \\
40-69 \\
41-70 \\
42-71 \\
43-72 \\
44-73 \\
45-74 \\
45-76 \\
46-77 \\
47-78 \\
48-79 \\
49-80 \\
50-82 \\
51-83 \\
52-84 \\
53-85 \\
53-86 \\
54-88 \\
55-89 \\
56-90 \\
57-91 \\
58-93 \\
59-94 \\
60-95 \\
61-96 \\
62-98 \\
63-99 \\
64-100 \\
65-101 \\
66-103 \\
67-104\end{array}$ \\
\hline
\end{tabular}

* Standardised method: EDTA anticoagulated but undiluted whole blood of haematourit of 0.35 or less

+ Working method: 4 vols EDTA blood plus 1 vol citrate diluent

The values incorporate a correction for dilution of blood by citrate in the working method. Proposed working method valid if The values incorporate a correction for dilu
$\mathbf{9 5} \%$ of results are within indicated limits. 
tation, method used for specimen mixing, volume of specimen, maintenance procedure and trouble shooting

7 Certificate of electrical conformity to a recognised standard (for example IEC 10:10:1: 1990).

\section{SAFETY ASSESSMENT}

1 Microbiological: to test for aerosol or droplet contamination during normal operation, one of the following two procedures, or an equivalent alternative, should be used:

(a) A series of tubes should be filled with a suspension of a marker organism (such as Serratia marcescens) and treated as blood specimens. Petri dishes containing nutrient agar are placed appropriately in the vicinity of the test system which is allowed to operate in its usual way. Petri dishes are then incubated and examined for growth of the marker organism.

(b) A few drops of a fluorescent chemical marker are added to blood samples which are then handled in the usual way for the ESR. Sheets of white absorbent paper are placed over possible areas of contamination in the vicinity of the test system. These are then examined under ultraviolet light for evidence of droplet contamination.

2 Mechanical: any potential hazard arising from the design of the test system and from any moving parts should be noted.

3 Waste disposal: any potential hazard (microbiological, chemical, or other) should be assessed.

\section{TECHNICAL ASSESSMENT}

Before the evaluation is formally started, the staff who will carry it out should have a preliminary period of training or familiarisation. This may include a pilot study.

1 Fresh human blood specimens are collected directly into the specified containers containing anticoagulant diluent, according to the manufacturer's instructions. Alternatively, blood can be collected into EDTA and subsequently diluted according to the manufacturer's instructions. Specimens should cover the range of results $15-105 \mathrm{~mm}$ with about the same number of specimens in each quartile. Blood for ESR tests should be stored at ambient temperature $\left(18-25^{\circ} \mathrm{C}\right)$ until tested and the tests should begin within 4 hours of collection. If the test system does not incorporate an automatic mixing device, the specimens should be mixed as specified for the standardised method: at least eight complete inversions $\left(180^{\circ} \times 2\right)$ for a $10-12 \mathrm{~mm}$ $\times 75 \mathrm{~mm}$ blood container and more inversions if the interior diameter of the container is smaller.

2 Precision should be based on replicate measurements (10 if possible) of a specimen from each quartile. The precision of the ICSH standardised (or reference) method should similarly be determined for comparative purposes.

3 Comparability between the test system and the ICSH standardised (or reference) method should be tested in parallel on at least
100 samples from patients with a wide variety of diseases and with ESR results distributed evenly in the range 15-105 $\mathrm{mm}$. Occasional blood samples fail to give a clear plasma-erythrocyte interface after sedimentation; if this occurs in either the test system or standardised (reference) method, the pair of values should be eliminated from the data set.

When blood specimens for the test system are diluted (4 vols blood plus 1 vol citrate), the undiluted ESR values for the ICSH standardised (or reference) method must be corrected for lack of dilution. The ICSH (1988) formula ${ }^{1}$ can be used for this purpose but verification of comparability of the test system may be determined with more accuracy over the range (15-105 $\mathrm{mm}$ ) of ESR values by using the table which already incorporates a correction for dilution. Validation is achieved if $95 \%$ of the test system results fall within the limits shown in the table. Use of the table, rather than the formula, is now recommended.

Paired results should be plotted on linear graph paper, with differences of the test system ESR from the ICSH standardised (or reference) ESR plotted on the vertical axis and the means of the two methods on the horizontal axis. $^{8}$

4 Sensitivity of response to added fibrinogen should be determined. A concentrated solution of fibrinogen of about $20 \mathrm{~g} / 1$ is made by dissolving human fibrinogen (such as Kabi Pharmaceuticals, Sweden) in distilled water. This is dialysed overnight against phosphate buffered saline (PBS; pH 7.4, normosmotic) to remove the high salt content. This is the stock fibrinogen solution whose fibrinogen concentration should be measured. To each of 5 aliquots of $5 \mathrm{ml}$ normal blood is added 1 $\mathrm{ml}$ of PBS alone, or mixtures of PBS and stock fibrinogen, equivalent to adding 0,5 , 10,15 and $20 \mathrm{mg}$ of fibrinogen.

Calculation of correlation coefficient and slope gives an assessment of linearity of response and sensitivity.

5 If the test system does not demand one specified specimen container, a comparison must be made between alternatives, including comparison of glass and plastic. Paired results on 20 tests should be analysed as above.

\section{EFFICIENCY ASSESSMENT}

1 The clarity, ease of reference, and comprehensiveness of the instruction manual should be evaluated.

2 Operational timing is established. This is based on a study carried out on a batch of at least 10 specimens and extends from specimen registration to result printout.

3 The level of training required by the operator should be assessed.

4 Reliability and maintenance: a written record is kept of any incidents during the period of evaluation, especially noting any "down time" due to failure of the test system.

5 Cost analysis should include capital cost over a nominal 5 year amortisation and cost of annual service/maintenance contract; consumables; and labour costs, taking account of 
the required seniority of the operator and the operational timing, as above. Financial comparison with the laboratory's current working method should be calculated on the basis of $10,30,50,100$, and 300 tests per day.

CONCLUSION OF EVALUATION

This should take account of the technical and functional reliability of the test system, current laboratory practice, the impact of the test system on the organisation of the laboratory and staff reaction to its introduction, and resource implications of the cost analysis described above.

\section{Summary}

The Expert Panel on Blood Rheology of the International Council for Standardization in Haematology (ICSH) has prepared new recommendations for measurement of the erythrocyte sedimentation rate (ESR) under the following categories:

1 ICSH reference method-ICSH now recognises, as its reference method for the ESR, the sedimentation of EDTA-anticoagulated but undiluted blood in traditional Westergren pipettes that meet ICSH specifications.

2 ICSH standardised method-ICSH recommends specifications for a new standardised method for the ESR based on the sedimentation of EDTA-anticoagulated, but undiluted blood in pipettes with a $200 \mathrm{~mm}$ scale and which are designed to avoid spillage of blood or aerosol generation. This standardised method may be used for verification or quality control of other ESR methods and, in future, may replace the reference method.

3 ICSH selected methods-ICSH recommends specifications for working methods, using diluted or undiluted blood, which may be considered as ICSH selected methods for routine use. A protocol is outlined for evaluation of such working methods against the ICSH reference method or the new ICSH standardised method.

Comments on these recommendations are invited and should be addressed to the ICSH Executive Secretary and to the Chairman of this panel.

1 International Committee for Standardization in Haematology (Expert Panel on Blood Rheology). Haematology (Expert Panel on Blood Rheology). Guidelines on selection of laboratory tests for monitor-
ing the acute phase response. $\mathcal{F}$ Clin Pathol 1988;41: ing the acut $1203-12$.

2 International Committee for Standardization in Haematology. Reference method for the erythrocyte sedimentation rate (ESR) test on human blood. $B r f$ Haematol 1973;24:671-3.

3 Fåhraeus R. The suspension-stability of the blood. Acta Med Scand 1921;55:1-228.

4 Westergren A. Studies of the suspension stability of the blood in pulmonary tuberculosis. Acta Med Scand 1921;54:247-82.

5 International Committee for Standardization in Haematology. Recommendation for measurement of erythrocyte sedimentation rate of human blood. $A \mathrm{~m} \mathcal{f}$ erythrocyte sedimentation
Clin Pathol 1977;68:505-7.

6 International Committee for Standardization in Haematology. Protocol for type testing equipment and apparatus used for haematological analysis. $\mathcal{f}$ Clin Pathol 1978;31:275-9.

7 International Federation of Clinical Chemistry (IFCC) and International Committee for Standardization in Haematology (ICSH). Approved recommendation (1986) on the theory of reference values. $₹$ Clin Chem Clin Biochem 1987;25:337-42.

8 Bland JM, Altman DG. Statistical methods for assessing agreement between two methods of clinical measurement. Lancet 1986;1:307-10. 


\section{Notices}

Association of Clinical Pathologists Junior Membership

Junior membership of the Association is available to medical practitioners who have been engaged in the practice of pathology for a period of less than four years. Junior members are able to remain in this category for a maximum of six years or on the attainment of consultant status. The annual subscription is $£ 34$ for those resident in the United Kingdom and $£ 65$ for those overseas. The annual subscription may be claimed against tax.

Junior members receive the fournal of Clinical Pathology each month. Other benefits are reduced registration fees to attend ACP scientific meetings, all the documents regularly sent to full members of the Association including $A C P$ News, which has a regular column for juniors, and the twice yearly summary of pathology courses included in the ACP programme of postgraduate education. Junior members have their own representative body, the Junior Members' Group, which has a direct input to Council.

For Junior Membership apply to: The Honorary Secretary, Association of Clinical Pathologists, 221 Preston Road, Brighton BN1 6SA. (0273) 561188

\section{THE EPIC STUDY}

The largest pan-European point prevalence study of nosocomial infection in intensive care units (ICUs), the EPIC Study, took place on the 29 April 1992 in 1472 ICUs throughout 17 western European countries. The initial data already provide an international overview of nosocomial infection in the ICU, and the comprehensive results of the 10 000-patient EPIC Study database were presented at the 13th International Symposium on Intensive Care and Emergency Medicine in Brussels, on 24 March 1993.

The EPIC Study results will be submitted for publication in an international journal later this year. Each participating ICU will receive an individual unit report followed by an advance copy of the special report on the European results of the study which will be available to non-participating ICUs in due course.

For further information on the EPIC Study final results meeting, please contact the EPIC Study Co-ordinator, Medical Action Communications, Action International House, Crabtree Office Village, Eversley Way, Thorpe, Egham, Surrey, TW20 8RY, UK.
Sport-The Health Challenges (in association with British Olympic Bid 2000)

Date

Wednesday 23 and

Thursday 24 June 1993

Venue

Manchester Conference Centre, UMIST, Sackville Street, Manchester M60 1QD

\section{Fee}

24 hour delegate/Full day delegate/single day delegate rates available from

The Royal Society of Health

Civic Reception and Delegate Dinner (optional)

All Enquiries to:

Miss Dawn Scanlan, Conference Department, The Royal Society of Health, 38A St George's Drive, London SW1V 4BH

Tel: 071-630 0121 Fax: 071-976 6847

\section{The Leeds Course in \\ Clinical Nutrition}

7-10 September 1993

St James's University Hospital, Leeds

Speakers include:

Dr T van der Poll (New York)

Professor B Rowlands (Belfast)

Professor N Wald (London)

Professor R C Coombes (London)

Professor J Garrow (London)

Further details from:

Mrs Hilary L Helme

Department of Continuing Professional Education

Continuing Education Building

Springfield Mount

Leeds LS2 9NG

Telephone (0532) 333233

19th Annual Lukes Conference:

Diagnostic Approaches to

Lymphoproliferative Disorders

Monday to Friday

October 15-19, 1993

Sponsored by Scripps Clinic and Research Foundation

This course is designed for haematologists, oncologists, and pathologists who are involved in the histological diagnosis or clinical evaluation of patients with malignancies of the lymphopoietic system. The conference will be held at the Sheraton Grande Torrey Pines Hotel in La Jolla, California. The course will offer 27 credit hours of Category I CME credit.

For further information contact: Department of Academic Affairs, 403C, Scripps Clinic and Research Foundation,

10666 N. Torrey Pines Road, La Jolla, CA 92037.

Telephone: (619) 554-8556 Fax: (619) 554-6310

\section{Lung Pathology}

London, National Heart and Lung Institute, 26-29 October, 1993

A comprehensive course of lectures and practical hands-on microscopy sessions aimed at pathologists in training and consultant pathologists wishing to update their knowledge of lung pathology. Places are limited to 24 .

\section{Fee $£ 220$ (or US\$360)}

Programme and application form from Professor B Corrin, Pathology,

Brompton Hospital,

London SW3 6NP, UK.

Fax 071-3518435

(44-71-351 8435 if outside UK).

\section{One-day Meeting on Gene Therapy}

\section{Royal Society of Medicine} Tuesday 1 June, 1993

Main topics:

Prospects for human gene therapy, advances in the application of gene therapy for SCID, cystic fibrosis, muscular disease, haemophilia, haematological diseases, cancer. Ethics, timing, implementation . . .

Organised by:

Section of Pathology of the RSM.

Registration Information:

Miss Judy Cook, Sections Office, RSM, 1 Wimpole Street, London W1M 8AE Fax: 0713553197

\section{Corrections}

Our apologies are extended to Drs Cooke, Jenkins and Stevens for duplication of text in their submissions to the Correspondence section of the Journal (Sentinel and Bactec blood culture systems; $\mathcal{F}$ Clin Pathol 1993;46:286.

We also apologise to Drs Cooke and Jenkins for the inadvertent inclusion of unnecessary text in Dr Stevens' reply.

Owing to printers' errors, incorrect formulae were published in the ICSM recommendations for measurement of erythrocyte sedimentation rate; $\mathcal{F}$ Clin Pathol 1993;46: 198-203. Diluted blood ESR $\mathrm{mm}=$ (undiluted blood ESR $\mathrm{mm} \times 0.86$ ) -12 is the correct version. Whenever this formula is repeated throughout the text the multiplication sign should be read as $\mathbf{x}$. A correct version of the recommendations has now been reprinted.

We apoligise to Professor Stuart for any inconvenience caused. 